\title{
Hospital Staff's Attitudes Toward and Knowledge About Dementia Before and After a Two-Day Dementia Training Program
}

\author{
Julia Schneider ${ }^{\mathrm{a}, *}$, Anton Schönstein ${ }^{\mathrm{a}}$, Winfried Teschauer ${ }^{\mathrm{b}}$, Andreas Kruse ${ }^{\mathrm{c}}$ and Birgit Teichmann ${ }^{\mathrm{a}}$ \\ ${ }^{a}$ Network Aging Research, Heidelberg University, Heidelberg, Germany \\ ${ }^{\mathrm{b}}$ German Alzheimer's Association, Bavaria, Nuremberg; Institute of Health, Bavarian Health \\ and Food Safety Authority, Nuremberg, Germany \\ ${ }^{\mathrm{c}}$ Institute of Gerontology, Heidelberg University, Heidelberg, Germany
}

\begin{abstract}
.
Background: The outcomes of hospitalized People with Dementia (PwD) are likely to be negative due to, among other key causes, negative staff attitudes and limited staff knowledge regarding dementia. Targeted interventions have been shown to positively change the attitudes of the hospital staff while also increasing their overall knowledge of dementia. However, training effects are often short-lived and frequently long-term effects are not examined in studies.

Objective: To examine whether attending a dementia training program changes the attitudes of hospital staff toward PwD and/or increases their knowledge levels about dementia, and whether or not these changes are stable.

Methods: The training program lasted two days and $N=60$ attending hospital staff members agreed to participate in the study. Data were assessed with questionnaires prior to the training, 3 months, and 6 months after the training. German versions of the Dementia Attitude Scale (DAS-D) and the Knowledge in Dementia (KIDE) scale were used. Additionally, data about perception of $\mathrm{PwD}$ and confidence in dealing with challenging behavior were collected and analyzed.

Results: After the training program, participants showed a significantly better attitude toward PwD as measured by DAS-D. These time-effects occurred in both DAS-D subscales ("dementia knowledge" and "social comfort"). Although a positive trend could be seen in the KIDE scale, no statistically significant increase occurred over time.

Conclusion: Specialist training programs seem to be promising in positively changing attitudes toward and increasing knowledge about PwD with long-term effects. Further research should address the effects of attitude change in patient care.
\end{abstract}

Keywords: Education, health facilities, health personnel, neurocognitive disorders, staff development

\section{INTRODUCTION}

The global aging population is increasing and as a consequence, the number of older multi-morbid and

\footnotetext{
${ }^{*}$ Correspondence to: Julia Schneider, Network Aging Research, Heidelberg University, Bergheimerstraße 20, 69115 Heidelberg, Germany. Tel.: +49 622154 8134; E-mail: schneider @ nar.uni-heidelberg.de.
}

frail patients treated in general hospitals rises [1]. In Germany, roughly 8.7 million patients over 65 years of age were treated in hospitals in 2016 [2]. People with Dementia (PwD) are an especially vulnerable subgroup of older patients in hospitals. Bickel et al. [3] estimated that in German hospitals at least $40 \%$ of patients aged 65 years and over have a mild cognitive impairment (MCI) or worse. Compared to people of similar age without dementia, PwD hospitalizations 
are not only longer [4] but their outcomes are also more likely to be negative [5]. Taking care of patients with cognitive impairment in the general hospital setting has been described as especially challenging for the responsible staff [6].

Surr et al. [7] highlighted stigmatization or negative staff attitudes and limited staff skills or knowledge regarding dementia, besides negative culture in wards and institutions and unsupportive physical environments, as key causes behind poor outcomes in PwD admitted to hospitals. In their recent review, Evripidou et al. [8] supported the assumption that acute care nurses' attitude toward PwD is rather negative. It was also found that hospital staff might not be aware of the needs that older people have, because they lack specialized education and understanding [4]. However, a higher dementia knowledge level is supposed to correlate positively with positive attitudes [8] and, vice versa, there is evidence that shows a correlation between lack of knowledge and negative attitudes toward older people [9]. The latest World Alzheimer Report [10] titled "Attitudes to dementia", highlights the importance of this topic in general.

Attitudes can be seen as a response to a person, object, or event that consists of three components: affect, behavior, and cognition [11]. It has been claimed that targeted interventions can positively change attitudes [12] and this is supported by studies that found positive changes in nurses' attitudes toward PwD after interventions [8]. Recent reviews show the growing global interest in dementia training programs for the hospital setting [13-15]. However, none of these reviews included a study from Germany, thus German data is missing in peer-reviewed journals covering this topic. The literature emphasizes that knowledge-based education alone will be less successful in improving care practice [16]. Dementia training programs have to be interactive and practically oriented [13, 17]. Furthermore, it ought to address affective and cognitive components of the learner so that they reflect on their behavior and the impact their care has on PwD [16].

Previous studies that measured attitude change or knowledge increase used various approaches. The best-known standardized tool to measure attitude toward PwD is the Approaches to Dementia Questionnaire (ADQ) [7, 18-20]. Almost all of the studies that used the ADQ could show significant improvements in hospital staff's attitudes toward PwD [7, $18,20]$. One of the standardized scales used in measuring knowledge gain is the Alzheimer's Disease
Knowledge Scale (ADKS) [19]. Given the fact that most validated dementia knowledge scales are unsuitable for dementia care in hospitals [21], Elvish et al. [22] developed and validated the Knowledge in Dementia (KIDE) scale for the hospital setting [23]. Several studies that used the KIDE Scale found significant increases in the dementia knowledge levels of hospital staff [20-23]. A known problem of training effects is that they are often short-lived and longterm effects are not examined in the aforementioned studies. Research with extended follow-ups is needed [15]. Due to the paucity of existing standardized tools in German language, the available tools of the Dementia Attitude Scale (DAS-D) and KIDE were utilized in the present study.

\section{Study aims}

This study aims to address the short-term and long-term changes in attitudes toward and knowledge about dementia following a two-day training course for the staff of a hospital association in Germany. Primary study questions were:

1. Do the attitudes of hospital staff toward PwD change after attending the training program "People with dementia in the general hospital" and can a long-term difference be observed over a period of 6 months?

2. Do the knowledge levels of hospital staff increase after attending the training program "People with dementia in the general hospital" and can a long-term difference be observed over a period of 6 months?

3. How strongly are the levels of knowledge regarding PwD and the attitudes toward PwD of German hospital staff correlated?

\section{METHODS}

The study protocol was approved by the ethics committee of the Faculty of Behavioral and Cultural Studies at Heidelberg University (Germany) (AZ Schn 2018 1/3).

\section{Settings and participants}

A hospital association located in the southeast of Germany consisting of six hospitals (one general hospital and five district hospitals) aimed to become dementia-friendly. The first step was to train their employees, starting with nurses and administrative 
staff working in emergency departments (EDs). The general hospital has $>500$ beds, whereas the size of the district hospitals ranges between 40 and 287 beds. The hospital association has six EDs (e.g., trauma surgical admission).

Dementia training sessions were obligatory for nurses working in EDs and hospital staff working in the administration of EDs. Nurses who had attended the further training course "emergency care" were not obliged to participate in the dementia training program. Due to low demand among employees, the training courses were also made available for interested employees of other wards or facilities (e.g., social service and geriatric wards).

In total, the capacities allowed for 140 employees to attend the training program and approximately this was the number of persons expected to participate in the study. To be included in this study the participants had to complete at least one day of the dementia training program "People with dementia in the general hospital" and had to give their written consent. Among the attendees, no further exclusion criteria were applied. Study participants were recruited before each training block: Prior to every training block, the study was orally introduced by the lecturer (W.T.) or one of the authors (J.S.), subsequently a letter of information describing the study-protocol was given to all training participants by the aforementioned persons.

\section{The dementia training program: "People with dementia in the general hospital"}

During the period from October 2018 to December 2018, six two-day training blocks on dementia were offered. Each day consisted of eight 45 minutes training sessions. Lessons were held in an educational center equipped for teaching. The group size varied between six and 16 persons. The training was conducted by an external lecturer from the "Alzheimer's Society of Bavaria" with profound theoretical knowledge about dementia and experience in teaching and science. A brief overview of contents is given in Table 1. Different types of delivering methods were used: teacher-centered teaching using Microsoft PowerPoint presentations, interactive exchanges between teacher and participants via verbal communication or using a flip chart, (educational) videos, individual- and partner work using worksheets, group discussions, as well as case studies. At the end of each day, a manual summarizing the key points of the training was given to all attendees.
Table 1

Overview of the training content [43]

\begin{tabular}{cc}
\hline Day 1: Knowledge and understanding \\
\hline Content & Current situation of PwD in the hospital \\
& Diagnostics and psychometric testing \\
& The disease dementia: clinical pictures, \\
causes, forms of dementia, \\
pharmacological therapy \\
Differentiation from other diseases: \\
depression and delirium \\
Understanding the symptoms and \\
challenging behavior \\
\\
\hline Day 2: Clinical skills \\
Concepts of non-pharmacological \\
interventions: person-centered care, \\
validation, communication \\
Dealing with challenging behavior \\
Milieu therapeutic interventions \\
Inclusion of relatives and biographical \\
knowledge
\end{tabular}

\section{Study design}

The analyzed data were part of a comprehensive mixed methods study including interviews and questionnaires. A single group repeated measures design was used to observe the training impact on staff and its consistency over a longer period of time. Questionnaire data were collected prior to the training (baseline) and follow-ups were conducted 3 months (follow-up 1), and 6 months (follow-up 2) after completion of the training.

\section{Outcome measures}

This article focuses on the information gathered by using questionnaires. The German version of the standardized DAS-D, translated and validated by Peng et al. [12], was used to assess staff attitudes toward PwD. This scale is based on the aforementioned tri-componential viewpoint of attitudes [24] and the 20-item scale consequently comprises affective, behavioral, and cognitive aspects. The scale has a two-factor structure labeled "social comfort" (e.g., "I am not very familiar with PwD") and "dementia knowledge" (e.g., "PwD like having familiar things nearby") $[12,24]$. The factor analysis of the original tool was performed on an American sample; however, it is assumed that there is closer cultural proximity to Switzerland. Therefore, the two-factor structure used for the present analysis is based on the results by Peng et al. [12]. The DAS-D statements are rated on a seven-point Likert scale ranging from 1 (strongly disagree) to 7 (strongly agree), with a 
higher score reflecting a more positive attitude. Cronbach's $\alpha$ for the entire sample was reported as 0.87 and 0.76 for the subgroup of the qualified health personnel [12]. Originally the term to address people with cognitive impairment in the questionnaire was people with "Alzheimer's disease and related dementias (ADRD)" [24]. We found this term difficult to understand and therefore used the German term for PwD.

Knowledge about dementia was measured with the German version of the KIDE scale [25]. The questionnaire consists of 16 dichotomous items where each correct answer yields one point. Consequently, total scores can range from 0 to 16 , higher scores indicating greater knowledge. The psychometric property of the English version was examined two times: the initial analysis reported a Cronbach's $\alpha$ value of 0.72 [22], the Cronbach's $\alpha$ value of the second analysis was 0.66 [23]. However, quality criteria for the German version are not yet sufficiently determined [26].

The following questions were asked at follow-up 1 and 2, respectively. Participants were asked in a closed-ended question whether their perceptions of PwD have changed since they attended the dementia training program. Possible responses were "yes: to the positive" or "yes: to the negative", "no", "maybe", and "I don't know". Afterwards, they were asked to describe in an open-ended question what exactly changed in their perceptions. Participants were also asked to rate their change in confidence in handling challenging behavior of PwD, which was measured using the difference of two six-point Likert scales with higher values indicating higher confidence.

\section{Data collection}

Participants had to sign a declaration of consent and were then asked to complete the baseline questionnaires, which they received from the lecturer prior to the training. To protect their identity, participants had to use a self-generated code on every questionnaire they received. All training participants received a training evaluation sheet immediately after each training block. Follow-up 1 and follow-up 2 questionnaires were sent by mail with an information letter, a handwritten thank-you card, and a pre-paid return envelope. Participants were asked to return the questionnaires within three weeks. All participants were sent a reminder card with an extended return deadline of two weeks. The period for answering the questionnaire was deliberately and generously chosen, in order to receive as many questionnaires as possible.

\section{Statistical analysis}

For descriptive statistics, means and standard deviations were calculated for continuous and approximately normally distributed variables, median and interquartile range for continuous or discrete but not normally distributed variables. Absolute and relative frequencies were calculated for the descriptive analysis of the categorical variables. Statistical analyses of the quantitative data were carried out with R [27] and SPSS V25.0 (IBM Corp, Armonk, New York). The changes of attitudes and knowledge over time were analyzed using mixed models with the R package "nlme" [28]. Initially, we tested the time-effect on the different outcomes as the only factorial predictor. In a second step, we included variables on prior work experience, years of school education, and interest in the training program. Mixed-effect model estimates were based on the restricted maximum likelihood method (REML). The within-group correlation structure of participants over time was set to "general" and therefore estimated from the data. This mixed-effects approach provides an effective way of handling missing data [29]. Furthermore, a bivariate Pearson correlation was calculated to examine the relationship between attitudes and knowledge. While a two-sided $\alpha$ of $5 \%$ was used throughout to mark statistical significance, this study was of exploratory nature and $\mathrm{p}$-values are therefore meant to be interpreted as descriptive only.

A quantitative content analysis [30] was conducted to analyze and quantify the open-ended question. An inductive category scheme was developed by one person (J.S.). After performing a test coding, the category scheme was further developed and all answers were then coded and analyzed in MAXQDA Analytics Pro 2018 (Release 18.2.3). To estimate the reliability, another researcher coded the answers. Cohen's Kappa was calculated for the statements of follow-up 1 and showed a substantial agreement of raters $(\kappa=0.62)$ [31]. Non-compliant segments were discussed and adjusted if necessary.

\section{RESULTS}

\section{Sample characteristics}

Although attendance was mandatory, few registered for participation. All staff who attended the dementia training program were invited to participate in the study. A total of $N=60$ out of 65 attending staff members gave their consent, took part in 
the study, and completed the baseline measures. The return rate, inclusion and exclusion of questionnaires at each stage are displayed in Supplementary Figure 1. Core characteristics of the sample at each point in time are reported in Table 2. Summarizing the main points, the majority of the original sample of participants were female $(n=55,91.7 \%)$ and the age ranges were mostly balanced, but only few participants were 56 years or older. Half of the participants $(n=31,51.7 \%)$ had at least 10 years, approximately one-fifth had at least $9(n=11,18.3 \%)$ and 12 years $(n=14,23.3 \%)$ of school education respectively. The participants were mainly registered nurses $(n=35,58.3 \%)$, medical assistants $(n=13$, $21.7 \%)$, or nursing assistants $(n=7,11.7 \%)$. Other professions taking part in the training program were geriatric nurses, sociologists, and educationalists. Most participants reported to work in wards or areas of EDs $(n=44,73.4 \%)$, while 9 participants $(15.0 \%)$ reported to work in geriatric wards. Some participants reported to work in a surgical day clinic, orthopedics, internal medicine, and sonography and functional diagnostics. The median working time of the sample was 8 years (IQR $=4.0-18.5$ ) working in hospitals in general and 3 years (IQR $=1.5-7.0)$ working in the current setting. While 42 participants $(70.0 \%)$ answered to work with PwD on a daily basis, 8 participants $(13.3 \%)$ reported to work with this patient group several times a week. However, only 2 participants $(3.3 \%)$ specified several times a month, 4 $(6.7 \%)$ mentioned less than once a month, and 4 participants $(6.7 \%)$ gave the answer not to know how often they work with PwD. Only 5 participants $(8.5 \%)$ had received prior training focusing on dementia. The prior received training ranged from 2 hours' duration to longer than a week. While 56 participants attended both training days completely, only 4 participants attended one complete training day.

\section{Dementia Attitudes Scale (DAS-D) and Knowledge in Dementia (KIDE) scale}

The descriptive analysis of the DAS-D results at baseline showed a mean value of 5.08 ranging from a min. of 3.45 to a max. of $6.65(S D=0.63)$. The subscale "social comfort" demonstrated a mean value of 4.80 (min. of 2.33; max. 6.75; $S D=0.83$ ), while the subscale "dementia knowledge" showed a mean value of 5.51 (min. of 3.38; max. 6.50; $S D=0.62$ ).

In the first step, mixed models were used to simply examine a possible time-effect in participants' attitudes after the training. There was a significant
Table 2

Descriptive statistics of the study sample at all three measurement times

\begin{tabular}{lccc}
\hline & $\mathrm{T} 0$ & $\mathrm{~T} 1$ & $\mathrm{~T} 2$ \\
\hline & $N=60(\%)$ & $n=37(\%)$ & $n=34(\%)$ \\
Gender & & & \\
$\quad$ Female & $55(91.7)$ & $35(94.6)$ & $32(94.1)$ \\
Age & & & \\
15-25 & $16(26.7)$ & $7(18.9)$ & $7(20.6)$ \\
26-35 & $17(28.3)$ & $9(24.3)$ & $8(23.5)$ \\
36-45 & $10(16.7)$ & $9(24.3)$ & $6(17.6)$ \\
46-55 & $12(20.0)$ & $9(24.3)$ & $10(29.4)$ \\
56-65 & $5(8.3)$ & $3(8.1)$ & $3(8.8)$ \\
Mother tongue & & & \\
German & $52(86.7)$ & $31(83.8)$ & $28(82.4)$ \\
Years of school (minimum) & & & \\
9y & $11(18.3)$ & $6(16.2)$ & $7(20.6)$ \\
10y & $31(51.7)$ & $18(48.6)$ & $16(47.1)$ \\
12y & $14(23.3)$ & $10(27.0)$ & $9(26.4)$ \\
Missing values & $4(6.7)$ & $3(8.1)$ & $2(5.9)$ \\
Professions & & & \\
Registered nurses & $35(58.3)$ & $24(64.9)$ & $22(64.7)$ \\
Medical assistants & $13(21.7)$ & $6(16.2)$ & $6(17.6)$ \\
Nursing assistants & $7(11.7)$ & $4(10.8)$ & $3(8.8)$ \\
Others & $4(6.7)$ & $3(8.1)$ & $3(8.7)$ \\
$\quad$ Missing value & $1(1.7)$ & - & - \\
Working area & & & \\
Emergency Department & $44(73.4)$ & $24(64.9)$ & $24(70.6)$ \\
Geriatric ward & $9(15.0)$ & $6(16.2)$ & $4(11.8)$ \\
Social service & $2(3.3)$ & $2(5.4)$ & $2(5.9)$ \\
Others & $4(6.7)$ & $4(10.8)$ & $4(11.8)$ \\
Not specified & $1(1.7)$ & $1(2.7)$ & - \\
\hline & & &
\end{tabular}

overall time-effect as measured by the DAS-D total scale $(F(2,126)=8.00, p<0.001)$. Further, a statistically significant effect could also be observed when both of the subscales "social comfort" $(F(2$, $126)=5.70, p=0.001)$ and "dementia knowledge" $(F(2,126)=5.46, p=0.005)$ were tested for timeeffects. Estimated time-effects for the DAS-D total scale and the associated subscales "social comfort" and "dementia knowledge" at follow-up 1 and followup 2 are displayed in Fig. 1.

In the second step we included potential covariates (interest in the training, prior work experience, and years of school education) into the analyses.

For the DAS-D total scale, when covariates were included into the analysis, the overall time-effect remained significant $(F(2,109)=6.33, p=0.003)$. Of the included covariates, only prior work experience had a negative significant effect $(F(2,109)=5.13$, $p=0.025$ ) on participants' attitudes. Effect estimates contrasting the follow-up groups to baseline, $\mathrm{p}$-values and 95\%-confidence intervals for both the "timeeffect-only" and the model incorporating covariates for the DAS-D total scale are reported in detail in Table 3. 
DAS-D Total

Time Effect for DAS-D

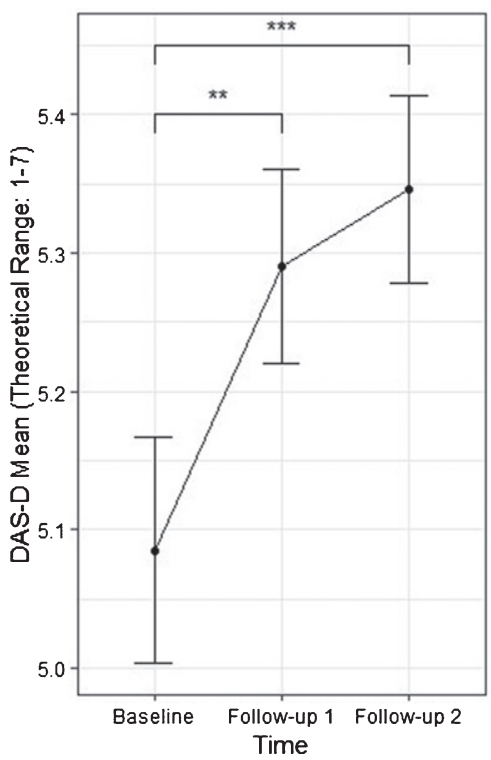

DAS-D Social Comfort

Time Effect for Social Comfort

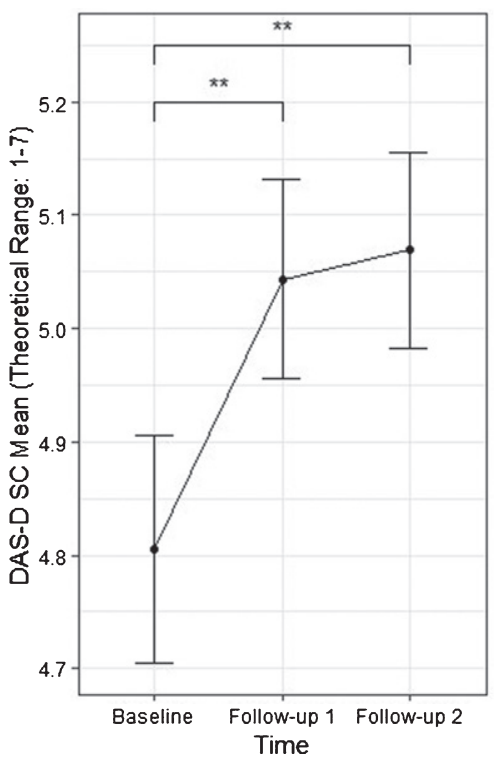

DAS-D Dementia Knowledge Time Effect for Dementia Knowledge

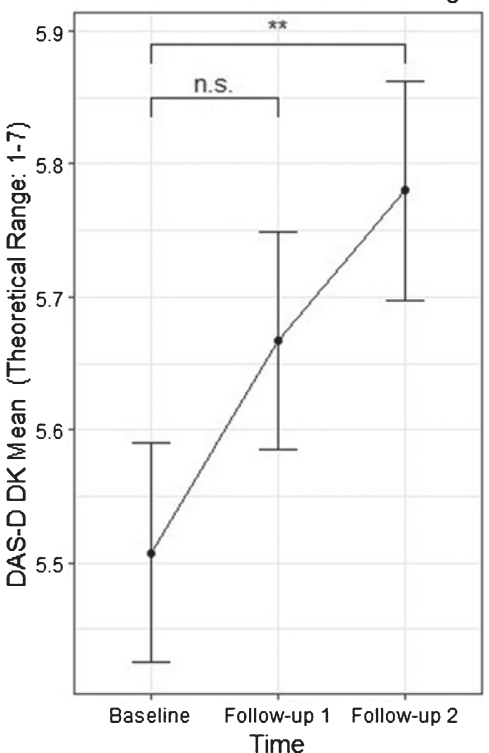

Fig. 1. Estimated time-effects for the DAS-D total scale and the associated subscales "social comfort" and "dementia knowledge" (** $p<0.01$, *** $p<0.001$ ) with a theoretical range from 1 to 7 . Whiskers represent standard errors.

Table 3

Multilevel models for the total DAS-D scale with time-effect and covariates

\begin{tabular}{|c|c|c|c|c|c|c|c|c|c|c|}
\hline & \multicolumn{5}{|c|}{ Model 1: Time-effect } & \multicolumn{5}{|c|}{ Model 2: Time-effect and covariates } \\
\hline & \multirow[t]{2}{*}{$b$} & \multirow[t]{2}{*}{$S E$} & \multirow[t]{2}{*}{$p$} & \multicolumn{2}{|c|}{$95 \% C I$} & \multirow[t]{2}{*}{$b$} & \multirow[t]{2}{*}{$S E$} & \multirow[t]{2}{*}{$p$} & \multicolumn{2}{|c|}{$95 \% C I$} \\
\hline & & & & Low & High & & & & Low & High \\
\hline Intercept & 5.08 & 0.08 & $<0.001^{* * *}$ & 4.92 & 5.25 & 5.06 & 0.32 & $<0.001^{* * *}$ & 4.42 & 5.70 \\
\hline \multicolumn{11}{|l|}{ Time } \\
\hline $\mathrm{T} 0^{b}$ versus $\mathrm{T} 1$ & 0.21 & 0.07 & $0.004^{* *}$ & 0.07 & 0.34 & 0.19 & 0.08 & $0.013^{*}$ & 0.04 & 0.34 \\
\hline $\mathrm{T} 0^{b}$ versus $\mathrm{T} 2$ & 0.26 & 0.07 & $<0.001^{* * *}$ & 0.13 & 0.40 & 0.26 & 0.07 & $<0.001^{* * *}$ & 0.11 & 0.40 \\
\hline Work experience (y) & . & . & . & . & . & -0.02 & 0.01 & $0.025^{*}$ & -0.03 & -0.00 \\
\hline Interest to participate ${ }^{a}$ & . & . & . & . & . & 0.05 & 0.03 & 0.080 & -0.01 & 0.11 \\
\hline \multicolumn{11}{|l|}{ Years of school } \\
\hline $9^{b}$ versus $10 \mathrm{y}$ & . & . & . & . & . & -0.17 & 0.22 & 0.438 & -0.60 & 0.26 \\
\hline $9^{b}$ versus 12 y & . & . & . & . & . & 0.13 & 0.24 & 0.585 & -0.34 & 0.61 \\
\hline
\end{tabular}

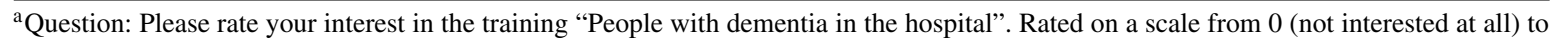
10 (very high interest). ${ }^{\mathrm{b}}$ Reference group. ${ }^{*} p<0.05,{ }^{* *} p<0.01,{ }^{* * *} p<0.001$.

Similar to the results of the total scale, for the DASD subscale "social comfort" the overall time-effect remained significant $(F(2,109)=5.49, p=0.005)$ when covariates were included into the analysis, and only prior work experience had a significant effect, which was negative $(F(1,109)=4.52, p=0.036)$. As with the total DAS-D scale, parameter details of the analysis with the subscale "social comfort", such as contrasts of baseline with follow-up 1 and follow-up 2 , are reported in Supplementary Table 1.

Lastly, the time-effect found for the DAS-D subscale "dementia knowledge" also remained significant $(F(2,109)=3.60, p=0.031)$ when covariates were included into the analysis. As opposed to the previously mentioned total DAS-D scale and the "social comfort", subscale not prior work experience $(F(1,109)=2.77, p=0.099)$, but interest in the training $(F(1,109)=5.82, p=0.018)$ and years of school education $(F(2,109)=4.81, p=0.010)$ were significant variables, with both of them having a positive association with "dementia knowledge". Parameter details for the analysis of the "dementia knowledge" subscale are reported in Supplementary Table 2.

The descriptive analysis of the KIDE results at baseline showed a mean value of 11.97 ranging from a min. of 7 to a max. of $15(S D=2.2)$. Further, possible time trends in knowledge about dementia were also examined using the KIDE scale. While the 
overall time-effect in this analysis was not significant $(F(2,128)=2.95, p=0.056)$, a positive trend could be observed (Supplementary Figure 2).

In order to examine the correlation between dementia knowledge and attitudes toward $\mathrm{PwD}$, a bivariate Pearson correlation with the baseline data from the total DAS-D scale and the KIDE scale was performed. A significant correlation with a medium effect size between the scales was found $(r(58)=0.36$; 95\% CI: $0.11-0.56, p=0.005)$.

\section{Perception of $P w D$ and dealing with challenging behavior}

Answers to the question as whether participants' perceptions of PwD have changed at follow-up 1 and 2 are reported in Fig. 2. At no measurement time did anyone respond "yes, to the negative". A slight decline in the positive change in perception is evident over time.

The analysis of the open-ended question at followup 1 indicated that participants reported to have a better understanding of PwD and their relatives. This category can be differentiated into having a better understanding of the disease and facts about dementia, and having a greater understanding of the patient's behavior and showing more compassion, the latter being the more frequently mentioned subcategory. The frequency of mentions of the two aforementioned categories is reversed at follow-up 2. The second most frequently mentioned category at follow-up 1 and 2 was recognizing needs of PwD associated with paying more attention to PwD, taking more time, with a change in the interaction with $\mathrm{PwD}$, and working practice. Occasionally participants reported to perceive that they know more about dementia, that they can identify PwD easier and also that the hospital is not an appropriate place for PwD.

In order to examine whether participants improved their clinical skills, they were asked to rate their confidence in dealing with challenging behavior before and after the training. At follow-up 1 (before the training: $M D=3.41, S D=1.10$; after the training: $M D=4.65, S D=0.81$ ) and follow-up 2 (before the training: $M D=3.56, S D=1.24$; after the training: $M D=4.82, S D=0.83$ ) respectively 34 participants answered. At both measurement time points, participants rated statistically significant to feel more confident in dealing with challenging behavior after attending the dementia training program (follow-up 1: $t(33)=8.426, p=<0.001$; follow-up 2: $t(33)=7.418, p=<0.001)$.

\section{DISCUSSION}

German data on this topic are rare in the international scientific exchange, and in addition, frequently long-term effects are not examined in dementia training studies [15]. The present study examines as a primary outcome long-term changes in attitudes toward and knowledge about dementia, following a two-day dementia training program for German hospital staff. The results indicate that the dementia training program "People with dementia in the general hospital" had a positive effect on the perceptions and attitudes of hospital staff toward PwD, increased participants' knowledge regarding $\mathrm{PwD}$, and confidence in handling challenging behavior. Long-term changes were observed over a period of 6 months after attending the dementia training program. Furthermore, the results indicate that a higher dementia knowledge level is connected to a more positive attitude toward PwD.

The baseline results showed a positive attitude toward PwD of the participants with potential for improvement. After the educational intervention, participants reported a more positive attitude toward PwD, which supports already existing literature stating, that education can effectively improve the attitude toward this vulnerable patient group [5, 7,

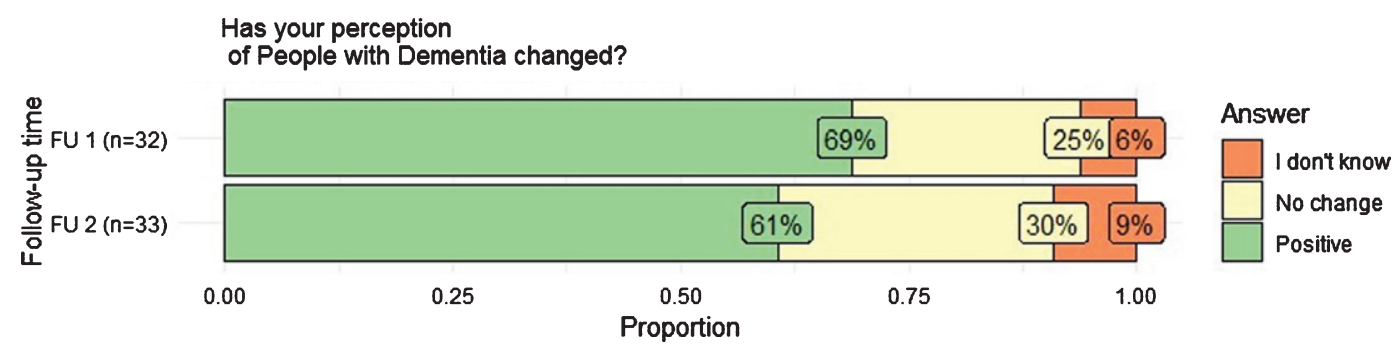

Fig. 2. Distribution of responses to follow-up 1 and 2. Possible responses were "yes: to the positive" or "yes: to the negative", "no", "maybe", and "I don't know". 
$8,18]$. Nevertheless, findings in other settings varied [32]. Longer work experience in hospitals had a negative impact on the attitude in general and in feelings of comfort around PwD, while none of the other control variables had a detectable impact on the entire DASD scale or its subscale "social comfort". Interestingly, Galvin et al. [33] noted that participants with the most prior experience in their sample did not show maintenance in gained knowledge and confidence. On the one hand, this could be due to the fact that the occupational education was carried out a long time ago and that opportunities for action were forgotten or were not sufficiently dealt with in the occupational education. This would strengthen the importance of further training courses. On the other hand, it may indicate that more experienced staff has been exposed to high burden for a long time, which can lead to "Coolout" in nursing practice $[34,35]$, and nurses may have made more negative experiences in dealing with $\mathrm{PwD}$.

Baseline results of the subscale "social comfort", which consists of affective and behavioral components [24], showed the highest potential for improvement. Personal involvement of $\mathrm{PwD}$ and/or relatives of PwD could help participants to develop supportive behaviors and pleasurable feelings of comfort around and in caring for PwD. Surr et al. [13] identified the direct involvement of PwD as a feature leading to more positive training outcomes summarizing video stories, vignettes, and personal involvement under this feature. However, personal contact might be more helpful.

While a statistically significant knowledge level increase occurred on the DAS-D subscale "dementia knowledge", this could not be shown on the KIDE scale, even though the trend was positive. However, a higher interest in the training program and a higher school education had an impact on the knowledge level regarding the DAS-D subscale "dementia knowledge". It may be possible that obligatory training does not have the desired effects [36] and higher qualified staff, with regard to their school education, learn easier and remember delivered training content better. Furthermore, persons with a higher interest in the training program may have had a higher intrinsic motivation, which leads to a deep approach in learning [37]. Nevertheless, it should be noted that the KIDE scale was developed to evaluate a different training program and the German version has yet to be validated. The results of the subscale "dementia knowledge", which comprises the cognitive component, are supported by the results of the positive changed perception of $\mathrm{PwD}$ which above all show a better and more understanding for PwD. While both aforementioned scales request declarative knowledge, participants seem also to have learned some practical knowledge or clinical skills. At follow-up 1 and follow-up 2, they report to feel more confident in dealing with challenging behavior. However, whereas this is an important finding for their daily work routine in caring for PwD and fostering feelings of comfort around PwD, it is not known whether this had, in fact, an effect on their working practice.

The assumption made in the literature that a positive attitude correlates with a higher dementia knowledge level [8] is strengthened by the findings of the present study where the correlation between the two standardized questionnaires had a positive effect of medium size. While this underscores the importance of education for hospital staff in general, the result is unsurprising given the cognitive component of attitudes [11] and the fact that the DAS-D scale operationalizes attitudes toward PwD in part through dementia knowledge.

However, the authors support the idea that single cause approaches with dementia training alone will not lead to sustainable changes in practice due to the complexity of the issue at hand. Instead, training courses should be part of an evidence-based intervention bundle, even though more research is needed to estimate their efficacy and to prove their effectiveness in practice [38]. Furthermore, a positive organizational and ward culture, a transformed care culture toward person-centered care and sustained action are needed [18, 33, 39].

No monetary incentive was offered to the participants for taking part in the study; however, researchers sent a handwritten card thanking them for their cooperation. The response rate for this study during follow-up $1(67 \%)$ and $2(68 \%)$ was relatively high in comparison to other studies conducted with similar topics. Galvin et al. [33] reported a response rate of $14 \%$, while Palmer et al. [40] reported a response rate of $25 \%$ for their questionnaires that were mailed out 3 months after completion of the training program. In addition, numbers of questionnaires sent out by email were not much higher $(27 \%)$ [41]. In the present study, only $62 \%$ of questionnaires during follow-up 1 and $57 \%$ during follow-up 2 could be included in the data analysis. It might be possible that mainly participants, that were, for example particularly interested in this topic returned followup 1 and 2 questionnaires. This should be taken into account when interpreting the results. 
The findings of this study are promising. Improvements appear relatively stable over time and significant results were verifiable 6 months after participants attended the dementia training program. Despite these results, the question remains as to whether or not an actual change in the behavior of hospital staff or their care for PwD took place. While the literature suggests that a higher knowledge level, increased confidence, and positive attitude are necessary to change staff behavior and improve patient outcomes $[15,42]$, behavioral data is needed to show how these changes may have influenced the quality of care of PwD.

\section{Limitations}

The present study has several limitations. Even though a larger sample size was expected, the actual number of study participants was small. Additionally, the study sample is not representative for the actual range of professions working in a hospital. In comparison to other studies conducted with similar topics, selective dropouts must also be taken into consideration, as a notable proportion of participants did not return their questionnaires. These aforementioned aspects may impact the generalizability of the study results. Larger randomized, stratified and importantly, controlled studies are necessary. The training was delivered by one person without a nursing background. Therefore, it is not known whether the impact of the dementia training program changes when other persons deliver the training and the environment changes. Regarding the scales, it should be mentioned that while the DAS-D scale was validated in a Swiss sample, the German version of the KIDE scale has yet to be validated. Furthermore, limitations of self-reported measures in general have to be taken into account, such as social desirability in the self-evaluation procedures. For this study, variables such as age, gender, profession, mother tongue, working area, previous dementia training, work experience in the current hospital ward, importance of the dementia training for their daily working routine, and frequency of contact and work with PwD were not included in the analysis due to restrictions caused by the sample sizes or their strong correlation with the included variables. Finally, the direct impact of attitude changes and knowledge increase on the patient outcome and participants practice was not observed and should be carried out by means of observational studies in the future. This is especially important to estimate what degree of change in attitude toward
PwD actually constitutes a practically meaningful as opposed to a solely statistically significant result.

\section{Conclusion}

Positive changes in attitudes and increase in knowledge regarding dementia were observed. These positive changes were demonstrated even 6 months after attending the training. Although these results are promising, they do not give insight into whether the participants' practices have changed and will influence the care of PwD or impact the patients' outcomes in any positive way. It has yet to be estimated how big the changes in attitude on the DAS-D scale or the increase in knowledge on the KIDE scale have to be to positively change participant's practice. Randomized controlled studies featuring observation of the staff's behavior could help to answer this question. Additionally, further development and validation of tools to measure attitude are needed.

\section{ACKNOWLEDGMENTS}

The authors thank all participating hospital staff, as well as the nursing directorate and their team involved in this study for their cooperation. The first author was reimbursed for travel costs by the clinic association for data collection and there are no further financial conflicts to report. Furthermore, the authors thank the members of the Graduate Program "People with Dementia in General Hospitals" for their valuable recommendations, and in particular Sebastian Ritzi, who supported the analysis of the qualitative data and enriched this work with his expertise.

J.S. and A.S. are members of the interdisciplinary Graduate Program "People with Dementia in General Hospitals", located at the Network Aging Research (NAR), Heidelberg University, Germany, and received a Doctoral Fellowship funded by the Robert Bosch Foundation, Stuttgart, Germany.

The entire project "People with dementia in the general hospital" of the German Alzheimer Association, Bavaria, which includes the dementia training program of the same name, is funded by the "Bavarian State Ministry of Health and Care". The lecturer of the dementia training program and a member of the "Alzheimer's Society of Bavaria" W.T. had no influence in the data collection and/or analysis to avoid conflict of interest and to report independent research.

Furthermore, it is explicitly pointed out that this study is not commissioned work. 
Authors' disclosures available online (https:// www.j-alz.com/manuscript-disclosures/20-0268r1).

\section{SUPPLEMENTARY MATERIAL}

The supplementary material is available in the electronic version of this article: https://dx.doi.org/ 10.3233/JAD-200268.

\section{REFERENCES}

[1] Reynish EL, Hapca SM, Souza N de, Cvoro V, Donnan PT, Guthrie B (2017) Epidemiology and outcomes of people with dementia, delirium, and unspecified cognitive impairment in the general hospital: Prospective cohort study of 10,014 admissions. BMC Med 15, 140.

[2] Statistisches Bundesamt (2017) Gesundheit. Diagnosedaten der Patienten und Patientinnen in Krankenhäusern (einschl. Sterbe- und Stundenfälle), 2016, https://www.destatis.de/ DE/Themen/Gesellschaft-Umwelt/Gesundheit/Krankenha euser/Publikationen/Downloads-Krankenhaeuser/diagnose daten-krankenhaus-2120621167004.pdf?__blob=publicatio nFile, Last updated March 1, 2018, Accessed February 7, 2020.

[3] Bickel H, Hendlmeier I, Heßler JB, Junge MN, LeonhardtAchilles S, Weber J, Schäufele M (2018) The prevalence of dementia and cognitive impairment in hospitals: Results from the General Hospital Study (GHoSt). Dtsch Arztebl Int 115, 733-740.

[4] Moyle W, Olorenshaw R, Wallis M, Borbasi S (2008) Best practice for the management of older people with dementia in the acute care setting: A review of the literature. Int $J$ Older People Nurs 3, 121-130.

[5] Dewing J, Dijk S (2016) What is the current state of care for older people with dementia in general hospitals? A literature review. Dementia (London) 15, 106-124.

[6] Hendlmeier I, Bickel H, Heßler-Kaufmann JB, Schäufele M (2019) Herausfordernde Pflegesituationen bei älteren Patienten im Allgemeinkrankenhaus: Der Einfluss kognitiver Beeinträchtigung und anderer patientenbezogener Faktoren. Z Gerontol Geriatr 52, 212-221.

[7] Surr CA, Smith SJ, Crossland J, Robins J (2016) Impact of a person-centred dementia care training programme on hospital staff attitudes, role efficacy and perceptions of caring for people with dementia: A repeated measures study. Int $J$ Nurs Stud 53, 144-151.

[8] Evripidou M, Charalambous A, Middleton N, Papastavrou E (2019) Nurses' knowledge and attitudes about dementia care: Systematic literature review. Perspect Psychiatr Care $\mathbf{5 5}, 48-60$.

[9] Hanson RM (2014) 'Is elderly care affected by nurse attitudes?' A systematic review. Br J Nurs 23, 225-229.

[10] Alzheimer's Disease International (2019) World Alzheimer Report 2019. Attitudes to dementia, https://www.alz.co. uk/research/WorldAlzheimerReport2019.pdf, Accessed December 18, 2019.

[11] Breckler SJ (1984) Empirical validation of affect, behavior, and cognition as distinct components of attitude. J Pers Soc Psychol 47, 1191-1205.

[12] Peng A, Moor C, Schelling HR (2011) Einstellungen zu Demenz. Übersetzung und Validierung eines Instruments zur Messung von Einstellungen gegenüber Demenz und demenzkranken Menschen (Teilprojekt 1), Universität Zürich, Zentrum für Gerontologie, http://www.zfg.uzh. $\mathrm{ch} /$ static/2011/Peng_Moor_Schelling_Einstellungen_Deme nz_2011.pdf, Accessed May 27, 2020.

[13] Surr CA, Gates C (2017) What works in delivering dementia education or training to hospital staff? A critical synthesis of the evidence. Int J Nurs Stud 75, 172-188.

[14] Surr CA, Gates C, Irving D, Oyebode J, Smith SJ, Parveen S, Drury M, Dennison A (2017) Effective dementia education and training for the health and social care workforce: A systematic review of the literature. Rev Educ Res 87, 9661002.

[15] Scerri A, Innes A, Scerri C (2017) Dementia training programmes for staff working in general hospital settings - a systematic review of the literature. Aging Ment Health 21, 783-796.

[16] Cowdell F (2010) The care of older people with dementia in acute hospitals. Int J Older People Nurs 5, 83-92.

[17] Schneider J, Gkioka M, Papagiannopoulos S, Moraitou D, Metz B, Tsolaki M, Kruse A, Teichmann B (2019) Expectations of nursing personnel and physicians on dementia training: A descriptive survey in general hospitals in Germany and Greece. Z Gerontol Geriatr 52, 249-257.

[18] Banks P, Waugh A, Henderson J, Sharp B, Brown M, Oliver J, Marland G (2014) Enriching the care of patients with dementia in acute settings? The Dementia Champions Programme in Scotland. Dementia (London) 13, 717-736.

[19] Smythe A, Jenkins C, Harries M, Atkins S, Miller J, Wright J, Wheeler N, Dee P, Bentham P, Oyebode J (2014) Evaluation of dementia training for staff in acute hospital settings. Nurs Older People 26, 18-24.

[20] Jack-Waugh A, Ritchie L, MacRae R (2018) Assessing the educational impact of the dementia champions programme in Scotland: Implications for evaluating professional dementia education. Nurse Educ Today 71, 205-210.

[21] Hobday JV, Gaugler JE, Mittelman MS (2017) Feasibility and utility of online dementia care training for hospital staff: The CARES ${ }^{\circledR}$ Dementia-Friendly Hospital ${ }^{\mathrm{TM}}$ program. Res Gerontol Nurs 10, 58-65.

[22] Elvish R, Burrow S, Cawley R, Harney K, Graham P, Pilling M, Gregory J, Roach P, Fossey J, Keady J (2014) 'Getting to Know Me': The development and evaluation of a training programme for enhancing skills in the care of people with dementia in general hospital settings. Aging Ment Health 18, 481-488.

[23] Elvish R, Burrow S, Cawley R, Harney K, Pilling M, Gregory J, Keady J (2018) 'Getting to Know Me': The second phase roll-out of a staff training programme for supporting people with dementia in general hospitals. Dementia (London) 17, 96-109.

[24] O'Connor ML, McFadden SH (2010) Development and psychometric validation of the Dementia Attitudes Scale. Int $J$ Alzheimers Dis 2010, 1-10.

[25] "Lern von mir". Unterstützung von Menschen mit Demenz in Allgemeinkrankenhäusern, "Lern von mir" Knowledge in Dementia Scale, Fachhochschule der Diakonie, https://www.fh-diakonie.de/obj/Bilder_und Dokumente/Lern_von_mir/Evaluationsblatt.pdf, Accessed September 27, 2019.

[26] Nienaber A, Volmar B, Wabnitz P, Löhr M (2015) Erste empirische Ergebnisse der wissenschaftlichen Begleitung des Projektes, Interdisziplinärer Demenzkoordinator. In: "Sprachen": Eine Herausforderung für die psychiatrische Pflege in Praxis - Management - Ausbildung Forschung, Schoppmann S, Stefan H, Hegedüs A, Fin- 
klenburg U, Needham I, Schulz M, Gurtner C, Hahn, S., eds., Vorträge, Workshops und Posterpräsentationen 12. Dreiländerkongress Pflege in der Psychiatrie in Wien, Forschung \& Entwicklung / Dienstleistung Pflege: Bern, pp. 148-151.

[27] R Core Team (2018) R: A language and environment for statistical computing. R Foundation for Statistical Computing, https://www.R-project.org/, Accessed May 27, 2020.

[28] Pinheiro J, Bates D, DebRoy S, Sarkar D, R Core Team (2019) nlme: Linear and nonlinear mixed effects models, R package version 3.1-143, https://CRAN.Rproject.org/package=nlme, Accessed May 27, 2020.

[29] Tabachnick BG, Fidell LS (2013) Using multivariate statistics, 6. ed., internat. ed., Always learning, Pearson, Boston, MA.

[30] Züll C (2015) Offene Fragen, GESIS - Leibniz-Institut für Sozialwissenschaften (GESIS Survey Guidelines), https://www.gesis.org/fileadmin/upload/SDMwiki/Offene Fragen_Zuell_08102015_1.1.pdf, Last updated January 2015, Accessed May 27, 2020.

[31] Landis JR, Koch GG (1977) The measurement of observer agreement for categorical data. Biometrics 33, 159-174.

[32] Spector A, Revolta C, Orrell M (2016) The impact of staff training on staff outcomes in dementia care: A systematic review. Int J Geriatr Psychiatry 31, 1172-1187.

[33] Galvin JE, Kuntemeier B, Al-Hammadi N, Germino J, Murphy-White M, McGillick J (2010) "Dementiafriendly hospitals: Care not crisis": An educational program designed to improve the care of the hospitalized patient with dementia. Alzheimer Dis Assoc Disord 24, 372-379.

[34] Kersting K (2016) Die "Coolout"-Theorie in der Pflegeausbildung: Neue Studien und Analysen, Mabuse, Frankfurt am Main.

[35] Bobbert M (2019) Berufliche Pflege und soziale Gerechtigkeit: Sechs sozialethische Problemanzeigen. Ethik Med 31, 289-303.
[36] Hicks WD, Klimoski RJ (1987) Entry into training programs and its effects on training outcomes: A field experiment. AMJ 30, 542-552.

[37] Marton F, Säljö R (2005) Approaches to learning. In: The experience of learning: Implications for teaching and studying in higher education, Marton F, Hounsell D, Entwistle N, eds., $3^{\text {rd }}$ (Internet) edition, University of Edinburgh, Centre for Teaching, Learning and Assessment: Edinburgh, pp. 3958.

[38] Sullivan DO, Mannix M, Timmons S (2017) Integrated care pathways and care bundles for dementia in acute care: Concept versus evidence. Am J Alzheimers Dis Other Demen 32, 189-193.

[39] Waugh A, Marland G, Henderson J, Robertson J, Wilson A (2011) Improving the care of people with dementia in hospital. Nurs Stand 25, 44-49.

[40] Palmer JL, Lach HW, McGillick J, Murphy-White M, Carroll MB, Armstrong JL (2014) The Dementia Friendly Hospital Initiative Education Program for acute care nurses and staff. J Contin Educ Nurs 45, 416-424.

[41] Sampson EL, Vickerstaff V, Lietz S, Orrell M (2017) Improving the care of people with dementia in general hospitals: Evaluation of a whole-system train-the-trainer model. Int Psychogeriatr 29, 605-614.

[42] Kang Y, Moyle W, Venturato L (2011) Korean nurses' attitudes towards older people with dementia in acute care settings. Int J Older People Nurs 6, 143-152.

[43] Fortbildungsprogramm. Kliniken und Rehabilitationseinrichtungen, Deutsche Alzheimer Gesellschaft Landesverband Bayern e.V. Selbsthilfe Demenz, https://www.alzheimer-bayern.de/images/downloads/wir fuer_sie/ehrenamtliche/broschuere_kliniken_internet.pdf, Accessed May 27, 2020. 